\title{
Advantages of Nuclear Fusion with Polarized Fuel
}

\author{
R. Engels*†, H.M. Awwad, K. Grigoryev and L. Huxold \\ Institute for Nuclear Physics, Research Center Jülich, 52428 Jülich, Germany \\ E-mail: r.w.engelsefz-juelich.de
}

\section{Büscher ${ }^{\dagger}$, I. Engin and A. Hützen}

Peter Grünberg Institute (PGI-6), Research Center Jülich, 52428 Jülich, Germany

${ }^{\dagger}$ also at Heinrich-Heine University Düsseldorf, Institute for Laser-and Plasma Physics, 40225

Düsseldorf, Germany

E-mail: m.buescherefz-juelich.de

\section{G. Ciullo}

Dipartimento di Fisica e Scienza della Terra, Università di Ferrara and INFN - sezione di Ferrara, Polo Scientifico e Tecnologico, 44122 Ferrara, Italy

E-mail: ciuldode.infn.it

V.D. Fotyev, K.A. Ivshin, E.N. Komarov, L.M. Kotchenda, P.V. Kravchenko, P.A. Kravtsov, S.G. Sherman, A.N. Soloviev, I.N. Soloviev, V.A. Trofimov, A.A. Vasilyev and M.E. Vznuzdaev

Petersburg Nuclear Physics Institute named by B.P. Konstantinov of National Research Centre

"Kurchatov Institute (PNPI)", 188300 Gatchina, Russia

E-Mail: kravchenko_pvepnpi.nrcki.ru

\section{Toporkov\#, I.A. Rachek and Yu.V. Shestakov\#}

Budker Institute of Nuclear Physics, 630090 Novosibirsk, Russia

\# also at Novosibirsk State University, 630090 Novosibirsk, Russia

E-mail: D.K.Toporkovdinp.nsk.su

\section{T.P. Rakitzis, D. Sofikitis, C.S. Kannis and G.K. Boulogiannis}

Department of Physics, University of Crete, 70013 Heraklion-Crete, Greece

Institute of Electronic Structure and Laser, Foundation for Research and Technology-Hellas,

71110 Heraklion-Crete, Greece

E-mail: ptrdies1.forth.gr

The use of nuclear polarized fuel, i.e. polarized $\mathrm{D}$, $\mathrm{T}$ or ${ }^{3} \mathrm{He}$, for coming fusion reactors promises to increase their energy output and to optimize the complete fusion process in various ways. But before these advantages can be utilized, several questions must be answered and technical issues must be overcome. Among others, the members of the PREFER collaboration started to investigate the different challenges of "polarized fusion".

XVII International Workshop on Polarized Sources, Targets \& Polarimetry

16-20 October 2017

Kaist, South Korea

\footnotetext{
* Speaker.

${ }^{\dagger}$ for the PREFER (Polarisation REsearch for Fusion Experiments and Reactors) Collaboration.
} 


\section{Introduction}

The idea of "polarized fusion" was first discussed by Maurice Goldhaber in 1954 [四]. The mirror reactions $\mathrm{t}+\mathrm{d} \rightarrow{ }^{4} \mathrm{He}+\mathrm{n}$ and ${ }^{3} \mathrm{He}+\mathrm{d} \rightarrow{ }^{4} \mathrm{He}+\mathrm{p}$ are theoretically understood since the 1960's. In 1970, a first experimental proof [ [ $]$ ] showed that the fusion cross sections for these reactions can be increased by a factor of 1.5 , when the nuclear spins of both projectiles are aligned. An increased cross section helps to raise the plasma temperature, and, therefore, the energy output is increased even further. The reason for the strong influence of the nuclear spin is that these s-wave dominated reactions are going through a $J=3 / 2^{+}$resonance in the intermediate nuclei ${ }^{5} \mathrm{He}$ or ${ }^{5} \mathrm{Li}$. This fusion channels are suppressed, if the spins of the reaction partners, $S=1$ for d and $S=1 / 2$ for $\mathrm{t}$ and ${ }^{3} \mathrm{He}$, are anti-parallel aligned. More details are shown in Fig.⿴囗十.

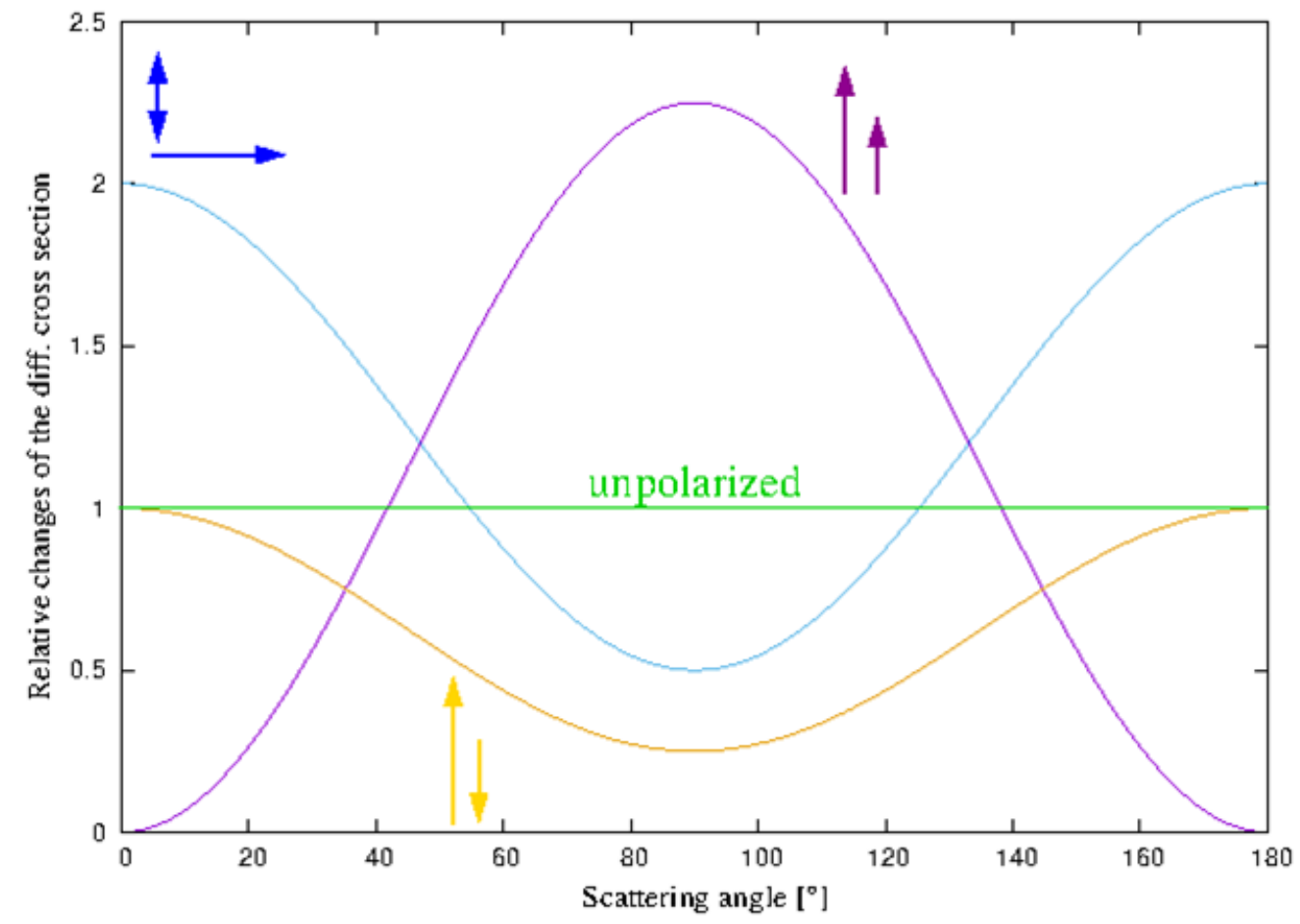

Figure 1: Relative difference of the polarized differential cross sections of the fusion reactions $\mathrm{t}+\mathrm{d} \rightarrow{ }^{4} \mathrm{He}$ $+\mathrm{n}$ or ${ }^{3} \mathrm{He}+\mathrm{d} \rightarrow{ }^{4} \mathrm{He}+\mathrm{p}$ at low energies for different spin combinations.

Due to the relatively long lifetime of the intermediate ${ }^{5} \mathrm{He}$ (or ${ }^{5} \mathrm{Li}$ ) nucleus of $0.3 \mathrm{~ns}$ [B] , the differential cross section in the cm-system does not depend on the scattering angle $\phi$ (green curve in Fig. 1). Even after transformation into the laboratory system the differential cross sections at the low resonance energies of $107 \mathrm{keV}(\mathrm{t}+\mathrm{d})$ and $430 \mathrm{keV}\left({ }^{3} \mathrm{He}+\mathrm{d}\right)$ do not depend much on the scattering angle of the projectiles.

If both spins are aligned parallel (purple), the differential cross section is suppressed for scattering angles around $\phi=0^{\circ}$ or $180^{\circ}$ and it is increased for $\phi=90^{\circ}$ by a factor of 2.25 . The total cross section is increased by the already mentioned factor of 1.5. If the nuclear spins are anti-parallel aligned (yellow curve) the differential cross section at $\phi=90^{\circ}$ is suppressed by a factor of four and 
the total cross section by a factor of two. When only the deuterons are negatively tensor-polarized, i.e. the polarization direction is perpendicular to the external magnetic field (blue curve), the total cross section is not changed, but the differential cross section is modified. This influence of the spins on the ejectile trajectories can be used to achieve further advantages: the neutron flux, e.g., can be directed towards the outer reactor wall, which would increase the tritium production via the $\mathrm{n}+{ }^{6} \mathrm{Li} \rightarrow{ }^{4} \mathrm{He}+\mathrm{t}$ reaction. In addition, the neutron flux to the inner blanket $\left(\phi \sim 90^{\circ}\right)$ would be decreased and, therefore, less cooling for the inner wall would be needed. The magnetic field coils could be positioned closer to the vacuum chamber, which would increase the magnetic field in the fusion plasma. This would help to enlarge the energy output $E$ of a fusion reactor, which depends on the magnetic field $B$ as $E \sim B^{6-8}$, where the exponent depends on the different reactor designs. Therefore, the choice of tensor-polarized $\mathrm{D}$ and unpolarized $\mathrm{T}$ as fuel for a Tokamak reactor allows one not only to optimize the energy production, but it may also be used to improve the T production inside the blanket.

\section{Challenges of "Polarized Fuel"}

The advantages of polarized fuel for energy production in future fusion reactors are impressive. Nevertheless, open questions must be answered and technical challenges have to be solved, before polarized fuel can become an option for more efficient fusion reactors.

\subsection{Production of Polarized Fuel}

The most important issue to solve is the production, handling and storage of the polarized fuel itself. The currently discussed approaches to feed a magnetic-confinement reactor with the necessary amount of fuel $\left(10^{22}-10^{23}\right.$ atoms/s) are to inject frozen fuel pellets or intense neutral particle beams in the $100 \mathrm{keV}$ energy range.

Up to now three different methods to polarize hydrogen atoms and its isotopes are used in nuclear and particle physics, e.g. to deliver polarized protons or deuterons for accelerator applications:

- Atomic beam sources (ABS)

Such sources, in principle modified Rabi setups, are installed at several accelerators to feed polarized internal targets like, e.g., that of the ANKE spectrometer in the storage ring COSY Jülich [䧃]. They use the focusing power of inhomogeneous magnetic sextupole fields to separate hydrogen atoms due to their electron spins (Stern-Gerlach effect). In combination with positive or negative ionization of the atoms they are also used as polarized ion sources [[]. The advantage of this method is the large polarization of $P=0.9$ and more, but the intensity of such sources is limited to about $10^{17}$ atoms/s, which is definitely not sufficient to feed a Tokamak.

- The "frozen spin" method

At large magnetic fields and very low temperatures the binding-energy differences of the hyperfine substates of atoms and molecules are maximized such that the Boltzmann distribution leads to an electron polarization of the atoms after a while that is subsequently transferred into a nuclear polarization. Compared to an ABS, this method is able to produce macroscopic amounts of polarized material, but this procedure can take up to several months. In 
addition, this method is technically very challenging: for HD molecules magnetic fields of, e.g., $17 \mathrm{~T}$ and temperatures of about $15 \mathrm{mK}$ are required to reach a nuclear polarization of the deuterons up to $P=0.5$. Nevertheless, this technique was and still is in use to serve as polarized target [6]. Due to the smaller magnetic moments of the deuteron, the hyperfine splitting energies for $\mathrm{D}_{2}$ molecules are even smaller and, therefore, nuclear polarization is not feasible up to now.

- Optical pumping with laser beams

The polarization of laser light can be used to polarize electrons of several gaseous atoms. Due to spin-exchange collisions and hyperfine interactions, the electron polarization can be transferred to the nucleons. This method has been used very successfully in the past 50 years for ${ }^{3} \mathrm{He}$ atoms and, nowadays, it is possible to deliver reasonable amounts of polarized atoms with a nuclear polarization of more than 0.8. Even for hydrogen, optical pumping was successful, e.g., at the polarized ion source at RHIC [四]. But "classical" optical pumping does not work for deuterium due to the larger amount of hyperfine substates and their very narrow energy levels.

Today, the production of polarized ${ }^{3} \mathrm{He}$ gas is not a problem anymore. Considering that optical pumping works for hydrogen atoms, it should also work for tritium, because the hyperfine splitting of both isotopes is identical in first order. What is missing, is the production of polarized deuterium in the necessary amounts for further tests. The PREFER collaboration (Polarization REsearch for Fusion Experiments and Reactors) is working on different concepts to solve this problem:

\section{Production and storage of polarized molecules}

Because of the small intensity of an ABS beam, it can never be used to feed a Tokamak for energy production. But, if the polarization of the atoms could be stored for one day, it might be possible to use them to feed a Tokamak at least for some seconds to measure the polarization lifetime in the plasma (see section 2.3). However, hydrogen atoms are radicals and cannot be stored without chemical reactions with the wall material or recombination of the atoms into molecules. Here, former measurements at polarized targets at NIKHEF [ [ $]$ ], IUCF [9] and HERMES [00] demonstrated that the nuclear polarization can be partially preserved during the recombination process on suitable wall materials. More dedicated experiments at the Research Center Jülich have shown that it is even possible to preserve the full polarization on special wall materials like Perfluorpolyether (PFPE). For molecules, the most powerful depolarization channels are the hyperfine beat of some substates at low magnetic fields and the interaction of the nuclear spin with the rotational magnetic moment $J$ of the molecules [ए]]. The hyperfine beat is suppressed by strong magnetic fields like those inside a Tokamak. Even the influence of the rotational magnetic moment $J$ can be suppressed by strong magnetic fields and disappears, when there is no rotation of the molecule anymore. For polarized hydrogen molecules $J=0$ is not possible due to symmetry reasons (orto-hydrogen with parallel spins must have an odd $J$ ), but it is allowed for polarized $\mathrm{D}_{2}$ or TD molecules. Therefore, if the polarized $\mathrm{D}_{2}$ or TD can be stored either as very cold gas or as ice, the atoms produced with an ABS might be collected to feed a Tokamak by frozen pellets or it can be used as a target for laser-induced fusion experiments [ए]]. 


\section{Filtering of polarized molecules: A molecular beam source (MBS)}

Like in an ABS, where the atoms are filtered due to their electron spins, the idea behind an MBS is to separate hydrogen/deuterium molecules due to their nuclear spin combinations. Of course, the magnetic moment of a proton is about 660 times smaller than that of the electron. To compensate the smaller focusing power of an inhomogeneous magnetic field, several effects can be used: instead of permanent magnets with a pole-tip field of $1.5 \mathrm{~T}$ like those of the permanent magnets inside an ABS, fields produced by superconducting coils can be 10 times stronger. In addition, these magnets can be about ten times longer and the velocity of the molecules can be decreased further compared to the atoms in an ABS. Last but not least another factor of two is won by the hydrogen molecule itself. The spins of the two protons can couple to a total spin and only the hyperfine substate, where both spins are parallel is important for polarized fuel. In a collaboration of the Budker Institute in Novosibirsk, the Research Center in Jülich and the Heinrich-Heine University in Düsseldorf, a proof-of-concept experiment is currently under preparation. The ABS in Novosibirsk is the only one worldwide that includes superconducting magnets and it can be modified for the filtering of polarized molecules. The molecules are sent through a ring-shaped nozzle, which can be cooled down to liquid helium temperature. Afterwards, they have to pass a corresponding aperture to build a hollow beam with a diameter that fits into the separating sextupole magnet. The molecules with positive spin projection along the magnetic field $\left(\left|I=2, m_{I}=2\right\rangle\right)$ are defocused and will hit the cooled wall, where they will freeze out as ice on the surface. The molecules with zero magnetic moment $\left(\left|I=0, m_{I}=0\right\rangle\right)$ will go straight on and hit the wall where they are lost, too. Even the tensor-polarized substates, e.g. $\left|I=2, m_{I}=0\right\rangle$, cannot escape from the magnet due to a corresponding aperture. Only the molecules with negative spin projection $\left(\left|I=2, m_{I}=-2\right\rangle\right)$ are deflected from the wall, can leave the magnet and are collected in a compression tube [[13]. A molecular beam intensity of about $10^{12}$ molecules/s behind this magnet can be reached due to the high magnetic field strength. In the next step a Lamb-shift polarimeter will be used to measure the polarization of these molecules. Of course, this intensity would not be sufficient to feed a Tokamak. Following the proof-of-principle with an existing setup, a dedicated apparatus of this type can be developed with, e.g., a much larger diameter of a multipole magnet to increase the production rate by orders of magnitude.

\section{A new method for optical pumping}

Beside the classical optical-pumping technique of hydrogen atoms, which does not work for deuterium up to now, there exists another option to produce nuclear polarized hydrogen or deuterium atoms. Sofikitis et al. describe in a recent publication [14] how to produce polarized deuterium atoms by UV dissociation of deuterium iodide molecules: a pulsed nozzle injects DI molecules into a vacuum chamber. A pulsed, circularly-polarized, UV laser at $266 \mathrm{~nm}$ excites the ${ }^{1} \Sigma_{0}$ ground state (with 0 electronic angular momentum projection along the DI bond) to the ${ }^{1} \Pi_{1}$ excited state (with an electronic angular momentum projection of +1 along the DI bond). The ${ }^{1} \Pi_{1}$ state correlates to atoms in the $\mathrm{m}$ states $\mathrm{I}(\mathrm{m}=3 / 2)$ and $\mathrm{D}(\mathrm{m}$ $=-1 / 2$ ), which sum up to 1 [ [U4]]. Therefore, the molecules dissociate and the electron spins of the deuterium atoms are polarized completely (for DI bonds aligned parallel to the laser 
polarization). The direction can be chosen by the polarization of the laser beam. This means that in this very short moment of about $0.1 \mathrm{~ns}$ only three hyperfine spin combinations are produced: $\left|m_{j}=1 / 2, m_{I}=1\right\rangle,|1 / 2,0\rangle$ and $|1 / 2,-1\rangle$. While $|1 / 2,1\rangle$ is a pure state the other states will show the hyperfine beat in phase, i.e. $|1 / 2,0\rangle$ will change into $|-1 / 2,1\rangle$ and back within $3 \mathrm{~ns}$, and the substate $|1 / 2,-1\rangle$ will move into $|-1 / 2,0\rangle$ and back. Therefore, the former electron polarization $P_{e}=+1$ and the deuteron polarization $P_{d}=0$ is transferred into $P_{e}=-1 / 3$ and $P_{d}=2 / 3$ after $1.5 \mathrm{~ns}$. If now the atoms are ionized, e.g. with another laser or with a pulsed electron beam, polarized deuterons are produced and can be accelerated further. For hydrogen and tritium a nuclear polarization of as much as $P_{p, t}=1$ is possible. A collaboration of the Research Center in Jülich, the Heinrich-Heine University in Düsseldorf and the University of Crete in Heraklion is planning a corresponding proof-of-principle experiment. Within these measurements it is foreseen that the polarized atoms are to be ionized at different magnetic fields to manipulate the hyperfine beat, i.e. the amplitudes of the hyperfine substates with non-parallel electron and nuclear spin. Afterwards, the polarization of the protons/deuterons will be measured with a Lamb-shift polarimeter.

Another option is the polarization of the rotational axis of $\mathrm{H}_{2}$ or $\mathrm{D}_{2}$ molecules (via Raman pumping) or DX molecules (via infrared excitation, where $\mathrm{X}$ is a halogen atom: $\mathrm{F}, \mathrm{Cl}, \mathrm{Br}$, I). Similar to the hyperfine beating, the rotational magnetic moment $J$ will interact with the nuclear spin and after some time of the order of $\mu \mathrm{s}$, the polarization of $J$ is transferred to a

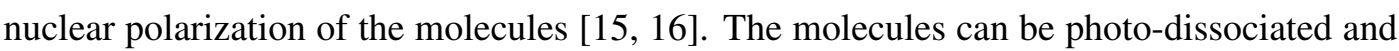
ionized, and the produced polarized proton or deuteron ions can be accelerated.

\subsection{The d-d double-polarized Cross Sections}

The aim of all experimental fusion reactors is to study the plasma confinement, the plasmawall interaction, and the different heating processes of the plasma. But the fusion processes $d+$ $\mathrm{t}$ or ${ }^{3} \mathrm{He}+\mathrm{d}$ are normally not investigated, because the tritium is radioactive. This complicates performing modifications, which are essential for the studies by experimental setups. Furthermore, the ${ }^{3} \mathrm{He}$ is very rare and expensive and the ${ }^{3} \mathrm{He}+\mathrm{d}$ reaction has the smallest cross section at low energies compared to the other reactions.

Therefore, the $\mathrm{d}+\mathrm{d} \rightarrow{ }^{3} \mathrm{He}+\mathrm{n}$ and $\mathrm{d}+\mathrm{d} \rightarrow \mathrm{t}+\mathrm{p}$ reactions are preferred to investigate the fusion processes, e.g., the energy transport from the plasma into the blanket with the help of the neutrons. But for these more complicated reactions (spin 1 on spin 1) with non-negligible p- and d-wave contributions, the influence of the nuclear spins are unknown up to now. However, this knowledge is absolutely necessary since these reactions will happen always in parallel to the "main" fusion reactions. In addition, a polarized d-d fusion plasma would be an efficient testing ground for further investigations of the spin influence (see section 2.3).

In the last 50 years several groups suggested to measure the necessary four analyzing powers and 16 spin-correlation coefficients at low energies down to $10 \mathrm{keV}$. But up to now only the analyzing powers down to $28 \mathrm{keV}$ are known [ㄱ, [8]]. Besides the necessity of an intense polarized beam in this energy range, a polarized target is also required. The relatively low cross sections at these beam energies below 1 mbarn and the maximum target thickness of a polarized jet target below $10^{12}$ atoms $/ \mathrm{cm}^{2}$ yield count rates between 10 and 100 counts/h only. 
A collaboration of the Petersburg Nuclear Physics Institute (PNPI) in Russia, the University of Ferrara (UNIFE) in Italy and the Institute for Nuclear Physics at the Research Center Jülich (IKP) in Germany is preparing the corresponding experimental setup at the PNPI [प्प]. An updated polarized deuteron source, formerly used at the Kernfysisch Versneller Instituut (KVI) at the University of Groningen in the Netherlands [[D], will deliver a polarized deuteron beam with enertgies up to 100 $\mathrm{keV}$ with a reasonable polarization of about $p_{z} \sim 0.7$. The polarized atomic beam source, producing the jet target, will be operated by the UNIFE. The IKP is responsible for the necessary Lambshift polarimeter [ [D] for the jet target and a nuclear reaction polarimeter [22] for the deuteron beam, based on the known analyzing powers of the d-d reactions. The interaction chamber and the detector system are built by the PNPI. The fact that in first order no background will reach the detectors allows to use rather cheap PIN photodiodes to detected the charged ejectiles, i.e. the protons, tritons and ${ }^{3} \mathrm{He}$ ions (see Fig.2).
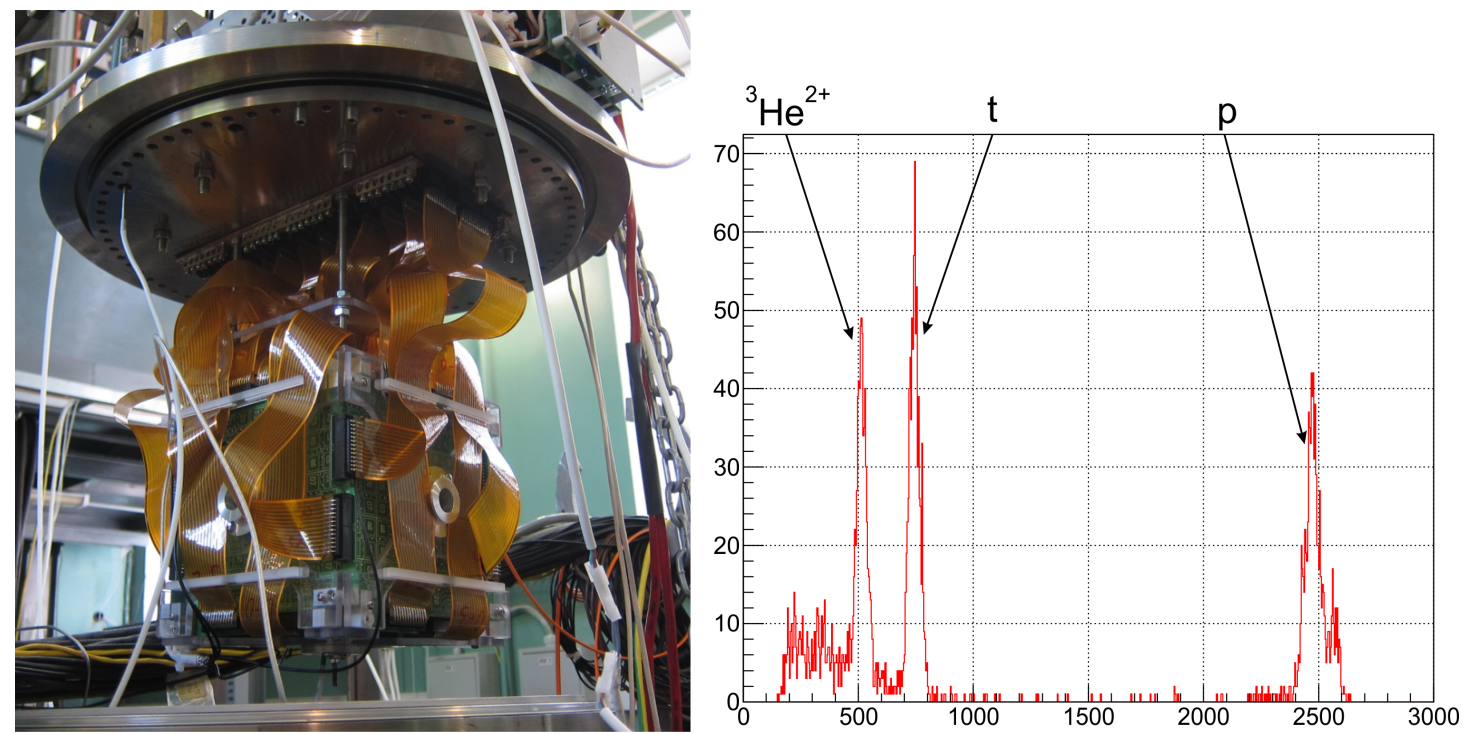

Figure 2: On the left: The cubic detector system that consists of about 300 PIN photodiodes around the interaction region of the crossed deuteron and atomic target beam. On the right: A typical spectrum obtained with the photodiodes during first tests with a deuterated titanium foil. The protons (3.02 MeV), tritons (1.01 $\mathrm{MeV})$ and ${ }^{3} \mathrm{He}$ ions $(0.82 \mathrm{MeV})$ of the $\mathrm{d}$-d fusion reactions can easily be separated and identified.

\subsection{Polarization Lifetime in Fusion Plasmas}

When the lifetime of the polarization in the fusion plasma is much shorter than the average time until the fuel nucleons need to fuse, the advantages of polarized fuel cannot be used. Kulsrud et al. [23] discussed in 1982 the different depolarization channels:

- recombination with unpolarized electrons in the plasma and the following hyperfine interaction,

- binary collisions and the following spin-spin or spin-orbit couplings,

- inhomogeneities of the magnetic field in the plasma, 
- resonant plasma waves,

- wall recycling of the plasma particles.

The outcome of their calculations was that the large magnetic fields of the confinement decrease the losses due to the different depolarization channels so that their influence should be negligible. Greenside et al. [24] contradicted the weak influence of the wall depolarization on most blanket materials, but at the same time they got a patent on wall coatings to avoid this depolarizing channel [25]. Coppy et al. [26] investigated the resonances of different plasma modes with the Larmor frequencies of the different ions. They come to the conclusion that depolarization due to this process is possible, but they turned the argument around: observing the depolarization would allow to analyze those plasma modes. Due to the different features of the existing or foreseen magnetic confinement reactors these calculations should be performed for each of them. Bartalucci [27] published his estimation for the ITER reactor in which the amount of wall interactions of the plasma particles will be significantly reduced, and for the planned IGNITOR reactor.

The polarization lifetime should be a minor problem for the different options for inertial fusion. Fusion processes with polarized deuteron beams are observed at several accelerators worldwide in

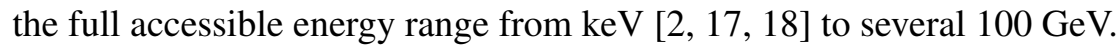

Following Temporal et al. [28], even for laser-induced nuclear fusion the polarization of the fuel can help to optimize the energy output or to reduce the necessary laser power. In this case, it is obvious that the laser-pulse duration of much less than one ns is too short to allow a reasonable depolarization by Larmor precession. But the extreme magnetic field amplitudes of the applied laser pulses in the order of $10 \mathrm{kT}$ might be the reason for other depolarization channels. Here, a coming measurement at the PHELIX laser in Darmstadt [29] should give a first answer: by laseracceleration of ${ }^{3} \mathrm{He}^{2+}$ ions from polarized ${ }^{3} \mathrm{He}$ atoms up to several $\mathrm{MeV}$, the preserved nuclear polarization of the ions will be observed with a nuclear reaction polarimeter based on the known asymmetries of the $\mathrm{d}\left({ }^{3} \mathrm{He},{ }^{4} \mathrm{He}\right) \mathrm{p}$ reaction in this energy range. If the accelerated ${ }^{3} \mathrm{He}^{2+}$ ions are still polarized after the laser-target interaction, then the polarization would survive the faster fusion processes, too.

A more direct experiment was supposed by Didelez and Deutsch [30]: when such a high-intensity laser beam hits polarized HD ice, several fusion processes will appear. The neutrons from the $d+$ $\mathrm{d} \rightarrow{ }^{3} \mathrm{He}+\mathrm{n}$ reaction can leave the interaction region easily and their count rate can be observed as a function of the HD polarization. But again, the spin-dependence of the $\mathrm{d}-\mathrm{d}$ reactions must be known (see section 2.1) before a polarization value of the deuterons during the fusion process can be calculated.

\section{Planned Experiments}

Recently, nuclear spin-polarized deuterium has been produced by the photodissociation of DI with circularly polarized light, at densities of at least $10^{19}$ deuterons $/ \mathrm{cm}^{3}$, and at a polarization of about $\mathrm{p}_{z}(\mathrm{D})=0.12$ (see sec. 2.1). Laser-fusion product-yield calculations have shown that these densities, combined with the NIF laser $\left(2 \mathrm{MJ} /\right.$ pulse) [B]] can produce about $10^{6}$ neutrons or protons from the d-t, $d^{3}{ }^{3} \mathrm{He}$, and $\mathrm{d}-\mathrm{d}$ reactions [14]]. Such a yield is sufficient to test the expected 
effects of polarized fusion for the first two reactions (particularly for $\mathrm{d}^{3} \mathrm{He}$, for which ${ }^{3} \mathrm{He}$ can be readily polarized to about $\left.\mathrm{p}_{z}\left({ }^{3} \mathrm{He}\right)=0.75\right)$, which are an increase in the reaction yield, and an anisotropic angular distribution. This observation would test whether the nuclear polarization survive the plasma long enough to benefit these fusion reactions. In addition, by aligning the DI bonds before the photodissociation, the deuteron nuclear polarization can be increased to about $\mathrm{p}_{z}(\mathrm{D})=$ 0.5. Such a large deuteron nuclear polarization could then, similarly, be used to measure the neutron angular distribution from the laser fusion of $d-d$ reactions, in order to help elucidate the effect of spin polarization in these reactions.

As described, even for magnetic-confined fusion in a Tokamak reactor the lifetime of the polarization is the crucial question. For this reason, Sandorfi et al. [32] are planning to feed the DIII-D Tokamak in San Diego with polarized ${ }^{3} \mathrm{He}$ and HD ice, normally used as polarized target for the CEBAF accelerator at the Jefferson Laboratories. Even when the production of polarized ${ }^{3} \mathrm{He}$ atoms by optical laser-pumping nowadays is not a problem any more, the polarized HD ice is made by the "brute force" method.

\section{Conclusion}

Nowadays, more than 50 years after the first ideas, "polarized fusion" seems to be within reach. The development of polarized ion sources and targets in the last decades opens the door for the production and storage of polarized fuel and for observing the necessary spin-correlation coefficients of the $d-d$ reactions at low energies. In the next step the preservation of the nuclear polarization in the different types of fusion plasmas, i.e. in magnetic confinement or in inertial fusion, can be investigated. The chance to increase the energy output of fusion reactors or to reduce the costs either by the increased cross sections or due to the control of the ejectiles cannot be ignored.

\section{Acknowledgments}

This work is partially supported by the Deutsche Forschungsgemeinschaft (grant no. BU 2227/1-1), the Russian Science Foundation (grant no. RSF 16-42-01009) and "Progetto Strategico" INFN-Energia.

\section{References}

[1] B.M. Schwarzschild, Physics Today 35 (1982) 17.

[2] Ch. Leemann et al.; Helv. Phys. Acta. 44 (1971) 141.

[3] G. Bohm et al.; Nucl. Phys. B 23 (1970) 93.

[4] M. Mikirtychyants et al.; Nucl. Instrum. Methods Phys. Res. Sect. A 721 (2013) 83.

[5] O. Felden, R. Gebel and R. Maier; Proc. $13^{\text {th }}$ Int. Workshop on Pol. Sources and Targets \& Polarimetry (PSTP 2009), Ferrara, Italy, World Sci. Pub., ISBN: 9789814462747 (2011) 23. 
[6] M.M. Lowry et al.; Proc. $9^{\text {th }}$ Int. Workshop on Pol. Sources and Targets \& Polarimetry (PSTP 2001), Nashville, USA, World Sci. Pub., ISBN: 9789812777683 (2001) 103.

[7] A. Zelenski et al., Proc. $15^{\text {th }}$ Int. Workshop on Pol. Sources and Targets \& Polarimetry (PSTP 2013), Charlottesville, USA, PoS (PSTP 2013) 048.

[8] J.F.J. van den Brand et al., Phys. Rev. Lett. 78 (1997) 1235.

[9] T. Wise et al., Phys. Rev. Lett. 87 (2001) 042701.

[10] P. Lenisa et al., Eur. Phys. J. D 29 (2004) 21.

[11] R. Engels et al.; Phys. Rev. Lett. 115 (2015) 113007.

[12] R. Engels et al.; contribution to this proceedings.

[13] D. Toporkov et al.; JETP Lett. 105 (2017) 289.

[14] D. Sofikitis et al.; Phys. Rev. Lett. 118 (2017) 233401.

[15] T.P. Rakitzis; Phys. Rev. Lett. 94 (2005) 83005.

[16] L. Rubio-Lago, D. Sofikitis, A. Koubenakis, T.P. Rakitzis; Phys. Rev. A 74 (2006) 042503.

[17] B. Becker et al.; Few-Body Syst. 13 (1992) 19.

[18] Y. Tagishi et al.; Phys. Rev. C 46 (1992) 46.

[19] A. Vasilyev et al., Nuclear Fusion with Polarized Fuel, Springer Proceedings in Physics 187 (2016) 35.

[20] H.R. Kremers and A.G. Drentje; AIP Conf. Proc. 421 (1998) 507.

[21] R. Engels et al.; Rev. Sci. Instr. 74 (2003) 4607.

[22] L. Kröll, A dd nuclear-reaction Polarimeter for the polarized Fusion Project, Diploma Thesis, Tech. University of Aachen (2010).

[23] R.M. Kulsrud et al.; Phys. Rev. Lett. 49 (1982) 1248.

[24] H.S. Greenside et al.; J. Vac. Sci. Technol. A 2 (1984) 619.

[25] US patent 4721595 from 26th of January 1988.

[26] B. Coppi et al.; Phys. Fluids 29 (1986) 4060.

[27] S. Bartalucci; Nuc. Inst. Meth. in Phys. Res. B 402 (2017) 240.

[28] M. Temporal et al.; Nucl. Fusion 52 (2012) 103011.

[29] I. Engin et al.; Nuclear Fusion with Polarized Fuel, Springer Proceedings in Physics 187 (2016) 55.

[30] J.P. Didelez and C. Deutsch; Laser Part. Beams 29 (2011) 169.

[31] https://lasers.llnl.gov

[32] A. Sandorfi et al.; Nuclear Fusion with Polarized Fuel, Springer Proceedings in Physics 187 (2016) 115. 